\title{
Gender Differences in Chronic Obstructive Pulmonary Disease Symptom Clusters
}

\author{
Haihong Zhang $\mathbb{D}^{\prime}$ \\ Fang $\mathrm{Wu}^{\mathrm{l}}$ \\ Hongli $\mathrm{Yi}^{2}$ \\ Dongjuan $\mathrm{Xu}\left(\mathbb{D}^{3}\right.$ \\ Nana Jiang ${ }^{4}$ \\ Yonghong $\mathrm{Li}^{5}$ \\ Ming $\mathrm{Li}^{\prime}$ \\ Kefang Wang'
}

'School of Nursing and Rehabilitation, Shandong University, Jinan City, Shandong Province, People's Republic of China; ${ }^{2}$ Department of Respiratory Medicine, Shandong Provincial Hospital, Jinan City, Shandong Province, People's Republic of China; ${ }^{3}$ School of Nursing, Purdue University, West Lafayette, IN, USA; ${ }^{4}$ Department of Respiratory Medicine, Dongfang Hospital Affiliated to Tongji University, Shanghai City, People's Republic of China; ${ }^{5}$ Department of Respiratory Medicine, Jinan Central Hospital, Jinan City, Shandong Province, People's Republic of China
Correspondence: Ming Li

School of Nursing and Rehabilitation, Shandong University, No.44 Wenhua Xi

Road, Lixia District, Jinan City, Shandong Province, 250012, People's Republic of China

Tel +86-53I-88382000

Email liming74@sdu.edu.cn
Purpose: Previous research has indicated that female and male patients may experience different levels of symptoms. However, no studies of chronic obstructive pulmonary disease (COPD) patients have compared the number and types of symptom clusters identified in male and female patients. Therefore, the purpose of this study was to investigate gender differences in symptom clusters among COPD patients.

Patients and Methods: A total of 371 eligible patients were enrolled in the study. We assessed nine COPD symptoms, namely, dyspnea, cough, sputum, chest tightness, sleep quality, fatigue, frailty, anxiety, and depression. Exploratory factor analyses were used to explore the underlying clusters of the COPD symptoms.

Results: Underlying the nine symptoms, female patients had 2 clusters, and male patients had 3 clusters. Specifically, the three general symptoms poor sleep, fatigue, and frailty loaded on the same symptom cluster with anxiety and depression in female patients, while the same 3 general symptoms loaded on the same symptom cluster with chest tightness and dyspnea in male patients. Moreover, cough and sputum not only were more common in male patients but also loaded together on a separate symptom cluster.

Conclusion: Our findings suggest that in order to improve fatigue, frailty, and poor sleep quality, symptom management strategies should more closely address anxiety and depression in female patients as well as chest tightness and dyspnea in male patients. Smoking cessation is particularly important in male COPD patients because they account for a much higher proportion of smokers and are more likely to have cough and sputum. These findings signify the importance of identifying and implementing gender-tailored symptom management strategies to relieve symptom burden in COPD patients to enhance their quality of life.

Keywords: quality of life, symptom burden, symptom management, factor analysis

\section{Introduction}

Chronic obstructive pulmonary disease (COPD) is a common, preventable, and treatable disease that is characterized by persistent respiratory symptoms and airflow limitations due to airway and/or alveolar abnormalities. It is usually caused by significant exposure to noxious particles or gases and is influenced by host factors, including abnormal lung development. ${ }^{1}$ At present, the global prevalence of COPD is $10.7 \%{ }^{2}$ In China, the latest research shows that the prevalence of COPD in adults aged 20 and over is $8.6 \%$ and among those over 40 , it is $13.7 \%$, while the prevalence in people over 60 is higher than $27 \%{ }^{3}$ The annual per patient hospitalization cost in China was reported as $\$ 1477.4$ Therefore, COPD has caused a substantial social-economic burden.

COPD imposes a high symptom burden on patients, especially those with severe disease. ${ }^{5}$ The burden is comparable to that of patients with cancer. ${ }^{6}$ COPD patients may 
suffer different symptoms, such as dyspnea, cough, sputum, chest tightness, poor sleep quality, fatigue, frailty, anxiety, and depression. $^{7}$ Two or more of these common symptoms may be related to each other and occur together, composing symptom clusters. Symptoms in a cluster are relatively independent of other clusters and may or may not have a common etiology or mechanism. Therefore, identifying symptom clusters is particularly important in guiding symptom assessment and management of COPD patients.

Previous research has indicated that female and male patients may experience different levels of symptoms. ${ }^{8}$ For example, female patients reported higher levels of dyspnea and anxiety than male patients. ${ }^{9}$ However, no studies of COPD patients have compared the number and types of symptom clusters identified in male and female patients. Therefore, the purpose of this study was to investigate gender differences in symptom clusters among COPD patients. Identifying symptom clusters in female and male patients separately may support the development of gender-tailored symptom management strategies to reduce symptom burden and improve quality of life.

\section{Patients and Methods}

\section{Study Design and Population}

We conducted a cross-sectional study from March to December 2019. Participants were recruited from 6 hospitals and 8 communities in Jinan City of Shandong Province, China. The inclusion criteria were as follows: (1) age $\geq 40$ years; (2) COPD as a primary diagnosis confirmed by a physician; (3) postbronchodilator $\mathrm{FEV}_{1} / \mathrm{FVC}$ of $<70 \%$ on pulmonary function test; and (4) willing to participate in the study and capable of understanding study questions. Participants were excluded if they had experienced exacerbation within the last 4 weeks, cancer, pneumonia, or major depressive disorder. This study was conducted in accordance with the amended Declaration of Helsinki and approved by the institutional review board of Shandong University (No. 2018-R-021). Written informed consent was obtained from each participant.

\section{Measures}

\section{Common COPD Symptoms}

We assessed nine COPD symptoms: dyspnea, cough, sputum, chest tightness, sleep quality, fatigue, frailty, anxiety, and depression. The modified Medical Research Council (mMRC) scale was used to assess the severity of dyspnea. ${ }^{10}$ Three items from the COPD Assessment Test (CAT) were used to assess the impact of cough, sputum, and chest tightness on a patient's life. ${ }^{11}$ Sleep quality was assessed by the Pittsburgh Sleep Quality Index (PSQI), which includes subjective sleep quality, latency, duration, efficiency, disturbances, use of sleep medications, and daytime dysfunction. ${ }^{12}$ The Manchester COPD Fatigue Scale (MCFS) incorporates physical, cognitive, and psychosocial domains to quantify the fatigue level. ${ }^{13}$ Frailty status was assessed using the multidimensional Edmonton Frail Scale (EFS) ${ }^{14}$ Anxiety and depression were measured by the brief Generalized Anxiety Disorder (GAD-2) ${ }^{15}$ and Patient Health Questionnaire (PHQ-2), ${ }^{16}$ respectively. The scales used to assess the above nine symptoms are the ones most used and validated in COPD patients. ${ }^{2,17-22}$ Higher scores represent more severe symptoms.

\section{COPD Stages}

Based on the $\mathrm{FEV}_{1}$ predicted value, the severity of COPD was categorized into four stages: mild $\left(\mathrm{FEV}_{1} \geq 80 \%\right)$, moderate $\left(\mathrm{FEV}_{1} 50-79 \%\right)$, severe $\left(\mathrm{FEV}_{1} 30-49 \%\right)$, and very severe $\left(\mathrm{FEV}_{1} \leq 30 \%\right)$.

\section{Exacerbation Rate}

The exacerbation rate was determined from self-reported episodes of worsening respiratory symptoms in the past 12 months. These increased COPD symptoms were beyond normal day-to-day variations and may require a change in antibiotics or steroids and/or access to health-care services (e.g., emergency department visits or hospitalization). ${ }^{23}$

\section{Other Patient Characteristics}

Sociodemographic variables included age, sex, race/ethnicity, marital status, and education years. Health-related variables included body mass index (BMI), smoking status, COPD duration, and the number of chronic conditions. The number of chronic conditions was assessed by the functional comorbidity index (FCI), which consists 18 conditions, counting their presence or absence. ${ }^{24}$ Physical activity was assessed using the International Physical Activity Questionnaire for the Elderly (IPAQ-E), which grouped patients into three levels: low, moderate, or high. ${ }^{25}$ Social support was measured by the social support rating scale (SSRS), which consists of subjective support, objective support, and the utilization of social support. ${ }^{26}$ A higher score indicates more social support.

\section{Statistical Analysis}

Continuous variables were summarized as the means and standard deviations (SDs), and categorical variables were summarized as frequencies and percentages. Gender 
differences were compared using independent samples t-tests or chi-square tests for continuous and categorical variables, respectively. Exploratory factor analyses (EFAs) were conducted to explore the underlying clusters of COPD symptoms. Principal-component factor models with maximum variance orthogonal rotation were used for EFA. When determining to which factor a symptom should be allocated, we used the largest factor loading criteria. Cronbach's alpha was calculated to evaluate the internal consistency of symptoms loaded on the same cluster. Alpha values of 0.8 or higher indicate good internal consistency. ${ }^{27}$ All analyses were performed using IBM SPSS statistics version 22.0 (IBM Corporation), and $\mathrm{P}$ values $<0.05$ were considered statistically significant.

\section{Results}

Table 1 presents the characteristics of the study population. A total of 371 eligible participants with COPD were enrolled in the study. Their mean age was 68 years (range 40-89). More than 64\% were men. There were no statistically significant differences between male and female COPD patients except in marital status, education years, and smoking status. A greater proportion of men were married than women ( $88 \%$ vs $64 \%$ ). On average, the men had received more years of education than the women. Nearly $87 \%$ of the male patients were current or former smokers. In contrast, fewer than $17 \%$ of the female patients were current or former smokers.

Table 2 shows the gender differences in the nine COPD symptoms. Compared to male COPD patients, female patients reported higher levels of anxiety, depression, and poor sleep, but the differences were not statistically significant. There were significant gender differences in cough and sputum symptoms, and male patients were more likely to have cough and sputum than females.

Table 3 presents the EFA results of symptom clusters for female and male COPD patients. Female patients had 2 clusters underlying the nine symptoms: mood-general symptom cluster (factor 1, including anxiety, depression, poor sleep, fatigue, and frailty) and respiratory symptom cluster (factor 2, including chest tightness, dyspnea, cough, and sputum). The two factors explained $60.9 \%$ of the variance in COPD symptoms in women, with eigenvalues of 3.75 and 1.73 for factor 1 and factor 2 , respectively. In contrast, male patients had 3 clusters underlying the 9 symptoms: respiratory-general symptoms cluster (factor 1, including chest tightness, dyspnea, poor sleep, fatigue, and frailty), mood symptom cluster (factor 2, including anxiety and depression), and cough-sputum symptom cluster (factor 3, including cough and sputum). The three factors explained $72.4 \%$ of the variance in COPD symptoms in men, with eigenvalues of $4.09,1.39$, and 1.04 for factors 1,2 , and 3, respectively. The signs of factor loading were consistent in each factor with loading scores greater than 0.3. The Cronbach's alpha for the 5 symptom clusters was $0.80,0.76,0.76,0.85$, and 0.83 , indicating good internal consistency.

\section{Discussion}

To the best of our knowledge, this is the first study to explore gender differences in symptom clusters among COPD patients. Underlying the nine symptoms, female patients had 2 clusters, and male patients had 3 clusters. Specifically, the three general symptoms poor sleep, fatigue, and frailty loaded on the same symptom cluster with anxiety and depression in female patients, while the same 3 general symptoms loaded on the same symptom cluster with chest tightness and dyspnea in male patients. Moreover, cough and sputum were not only more common in male patients but also loaded together on a separate symptom cluster.

As expected, poor sleep, fatigue, and frailty loaded together regardless of gender. Consistent with previous studies, ${ }^{28-30}$ the three symptoms were correlated with each other. Poor sleep, fatigue, and frailty are all increasingly common with advancing age. ${ }^{31-33}$ Fatigue is a manifestation of sleep disorders among the middle-aged sleep clinic population. ${ }^{34}$ Poor sleep negatively affects hormonal and metabolic functions, reduces growth hormone secretion and promotes cortisol secretion. ${ }^{35}$ Lower growth hormone levels contribute to a decline in muscular mass and strength. ${ }^{36}$ Sarcopenia in COPD patients is often considered to have a remarkable overlap with frailty. ${ }^{37}$ As these conditions are also involved in the pathophysiological mechanisms of fatigue and frailty, the association between poor sleep, fatigue and frailty is not an unexpected outcome.

However, our findings indicate that the three general symptoms loaded together with anxiety and depression in female patients, while they loaded together with chest tightness and dyspnea in male patients. When female patients experience stress, they may react to it with anxiety and depression, as well as a variety of general symptoms, such as poor sleep, fatigue, and frailty. This finding suggests that addressing anxiety and depression is particularly important for female patients because it may help relieve their feelings of fatigue and frailty and improve their sleep quality. In contrast, in male 
Table I Characteristic of Participates

\begin{tabular}{|c|c|c|c|c|}
\hline \multirow[t]{3}{*}{ Characteristics } & All & Female & Male & \multirow[t]{3}{*}{$P$ value } \\
\hline & No.(\%) or Mean \pm SD & No.(\%) or Mean \pm SD & No.(\%) or Mean \pm SD & \\
\hline & $(n=37 I)$ & $(n=133)$ & $(n=238)$ & \\
\hline \multicolumn{5}{|l|}{ Gender } \\
\hline Female & $133(35.8 \%)$ & & & \\
\hline Male & $238(64.2 \%)$ & & & \\
\hline Age, year & $68.09 \pm 9.44$ & $68.05 \pm 9.91$ & $68.11 \pm 9.18$ & 0.956 \\
\hline \multicolumn{5}{|l|}{ Race/ethnicity } \\
\hline Han & 357 (96.2\%) & $128(96.2 \%)$ & 229 (96.2\%) & 0.616 \\
\hline Other & $14(3.8 \%)$ & $5(3.8 \%)$ & $9(3.8 \%)$ & \\
\hline \multicolumn{5}{|l|}{ Marital status } \\
\hline Married & 294 (79.2\%) & 85 (63.9\%) & $209(87.8 \%)$ & $<0.001$ \\
\hline Single/divorced/widowed & 77 (20.8\%) & $48(36.1 \%)$ & 29 (I2.2\%) & \\
\hline Education, year & $7.57 \pm 4.03$ & $6.59 \pm 4.31$ & $8.12 \pm 3.76$ & $<0.001$ \\
\hline Health insurance & & & & 0.512 \\
\hline Yes & $364(98.1)$ & $|3|(98.5)$ & $233(97.9$ & \\
\hline No & $7(1.9)$ & $2(1.5)$ & $5(2.1)$ & \\
\hline BMI, kg/m² & $24.95 \pm 6.78$ & $24.83 \pm 4.67$ & $25.02 \pm 7.72$ & 0.795 \\
\hline COPD duration, year & $13.01 \pm 13.29$ & $14.08 \pm 14.82$ & $12.4 \mid \pm 12.35$ & 0.246 \\
\hline \multicolumn{5}{|l|}{ Severity of COPD } \\
\hline Mild & 35 (9.5\%) & $8(6.0 \%)$ & 27 (II.4\%) & 0.169 \\
\hline Moderate & $114(30.8 \%)$ & $40(30.1 \%)$ & $74(31.2 \%)$ & \\
\hline Severe & 115 (3I.1\%) & 49 (36.8\%) & $66(27.8 \%)$ & \\
\hline Very severe & $106(28.6 \%)$ & $36(27.1 \%)$ & $70(29.5 \%)$ & \\
\hline \multicolumn{5}{|l|}{ Exacerbation rate } \\
\hline$<2 /$ year & $250(67.4 \%)$ & 91 (68.4\%) & $159(66.8 \%)$ & 0.421 \\
\hline$\geq 2 /$ year & 121 (32.6\%) & $42(31.6 \%)$ & 79 (33.2\%) & \\
\hline \multicolumn{5}{|l|}{ Smoking status } \\
\hline Current smoker & $82(22.1 \%)$ & $9(6.8 \%)$ & $73(30.7 \%)$ & $<0.001$ \\
\hline Former smoker & $146(39.4 \%)$ & $13(9.8 \%)$ & $133(55.9 \%)$ & \\
\hline Never smoker & 143 (38.5\%) & III (83.5\%) & $32(13.4 \%)$ & \\
\hline Number of chronic conditions & $1.43 \pm 1.42$ & $1.53 \pm 1.46$ & $1.37 \pm 1.40$ & 0.277 \\
\hline \multicolumn{5}{|l|}{ Physical activity } \\
\hline Low & 140 (37.7\%) & 48 (36.1\%) & $92(38.7 \%)$ & 0.811 \\
\hline Moderate & $173(46.6 \%)$ & 65 (48.9\%) & $108(45.4 \%)$ & \\
\hline High & $58(15.6 \%)$ & $20(15.0 \%)$ & $38(16.0 \%)$ & \\
\hline Social support & $37.53 \pm 7.68$ & $37.38 \pm 8.26$ & $37.61 \pm 7.36$ & 0.780 \\
\hline
\end{tabular}

Note: We used mean and standard deviation (SD) to describe continuous variables and frequency and percentage to describe categorical variables.

patients, poor sleep, fatigue, and frailty were closely related to chest tightness and dyspnea. Strategies to manage chest tightness and dyspnea in male patients may help improve their general symptoms. Our study provides insights regarding gender-specific interventions in clinical practice and the potential benefits of considering gender-tailored symptom management.

We also found that cough and sputum loaded together on a separate symptom cluster in male patients, and they were more likely to have cough and sputum. This may be 
Table 2 Comparison of Patient Symptoms by Gender (Mean \pm SD)

\begin{tabular}{|l|c|c|c|c|}
\hline \multirow{2}{*}{ Symptoms } & All & Female & Male & \multirow{2}{*}{ P value } \\
\cline { 2 - 4 } & $\mathbf{( n = 3 7 I )}$ & $\mathbf{( n = 1 3 3 )}$ & $\mathbf{( n = 2 3 8 )}$ & \\
\hline Anxiety & $1.43 \pm 1.74$ & $1.58 \pm 1.72$ & $1.34 \pm 1.74$ & 0.205 \\
Depression & $1.38 \pm 1.62$ & $1.47 \pm 1.65$ & $1.32 \pm 1.60$ & 0.392 \\
Poor sleep & $9.00 \pm 3.46$ & $9.26 \pm 3.57$ & $8.85 \pm 3.40$ & 0.274 \\
Fatigue & $17.32 \pm 11.91$ & $15.91 \pm 11.00$ & $18.11 \pm 12.35$ & 0.089 \\
Frailty & $5.23 \pm 2.83$ & $5.03 \pm 2.62$ & $5.35 \pm 2.93$ & 0.298 \\
Chest tightness & $1.93 \pm 1.55$ & $1.91 \pm 1.46$ & $1.95 \pm 1.61$ & 0.833 \\
Dyspnea & $1.85 \pm 1.15$ & $1.75 \pm 1.12$ & $1.91 \pm 1.16$ & 0.210 \\
Cough & $1.92 \pm 1.36$ & $1.71 \pm 1.35$ & $2.03 \pm 1.35$ & 0.026 \\
Sputum & $1.96 \pm 1.32$ & $1.62 \pm 1.28$ & $2.15 \pm 1.30$ & $<0.001$ \\
\hline
\end{tabular}

Abbreviation: SD, standard deviation.

explained by the gender differences in smoking. There was a significantly higher proportion of current or former smokers among the male patients than among the female patients (current smoker: $31 \%$ vs $7 \%$; former smoker: $56 \%$ vs $10 \%$ ). Previous studies have indicated that COPD patients who were smokers had more symptoms of chronic cough and sputum than non-smokers. ${ }^{38-40}$ This also highlights the importance of quitting smoking Smoking cessation not only prevents COPD development but also slows COPD progression, regardless of severity.

This study has several limitations. First, the COPD patients were recruited from one city using a convenience sampling method. Convenience sampling is less likely than probability sampling to produce representative samples. In our sample, there was a greater proportion of males and smoking was much higher among males. Therefore, the generalizability of our findings is limited. Second, the symptoms were based on self-reporting and thus are subject to bias. Third, we assessed symptoms at one time point in this cross-sectional study; thus, responses may not reflect the stability of these symptom clusters over time. Fourth, marital status, years of education and smoking rate were statistically significant for men and women. We discussed the gender differences in smoking rate which may explain our findings. Marital status and education may have played a role in our findings, as well as other omitted potentially confounding factors such as phenotypes or traits of COPD. For example, different phenotypes or traits of COPD may have different symptoms manifestation. Probability sampling with larger sample size and more detailed information on phenotypes or traits of COPD, lung functions, and related inflammatory parameters would be beneficial for further examining whether the symptom clusters exhibit the same gender patterns among COPD patients. Despite these limitations, our study has scientific values. It focused on a research question which was not commonly investigated. Our results give new insights into COPD patients' symptoms and gender differences, which not only set a new direction for further research but also signifies the need for a new look at this aspect of COPD in everyday clinical practice.

\section{Conclusion}

Using a large sample of COPD patients, this study addressed knowledge gaps regarding gender differences in the number and types of symptom clusters. Our findings suggest that to improve fatigue, frailty, and poor sleep quality, symptom management strategies should more closely address anxiety and depression in female patients and chest tightness and dyspnea in male patients. Smoking cessation is particularly important in male COPD patients because they include a much higher proportion of smokers and are more likely to have cough and sputum. These findings signify the importance of identifying and implementing gender-tailored

Table 3 Gender Differences in Symptom Clusters: Results of Exploratory Factor Analysis

\begin{tabular}{|l|c|c|c|c|c|}
\hline \multirow{2}{*}{ Symptoms } & \multicolumn{2}{|c|}{ Female } & \multicolumn{2}{c|}{ Male } \\
\cline { 2 - 6 } & Factor I & Factor 2 & Factor I & Factor 2 & Mood \\
\cline { 2 - 6 } & Mood- General & Respiratory & Respiratory-General & 0.917 & \\
\hline Anxiety & 0.809 & & & 0.874 \\
Depression & 0.783 & & & \\
Poor sleep & 0.642 & & 0.379 & \\
Fatigue & 0.736 & & 0.729 & \\
Frailty & 0.688 & 0.744 & 0.729 & \\
Chest tightness & & 0.654 & 0.724 & \\
Dyspnea & & 0.769 & 0.856 & & \\
Cough & & 0.833 & & 0.920 \\
Sputum & & & & \\
\hline
\end{tabular}


symptom management strategies and relieve symptom burden in COPD patients to enhance their quality of life.

\section{Acknowledgments}

The study was supported by the Chinese Nursing Society (ZHKY201824).

\section{Disclosure}

The authors report no conflicts of interest with regard to this work.

\section{References}

1. Global strategy for the diagnosis, management, and prevention of chronic obstructive pulmonary disease (2020 REPORT). Available from: www.goldcopd.org. Accessed March 31, 2021.

2. Adeloye D, Chua S, Lee C, et al. Global and regional estimates of COPD prevalence: systematic review and meta-analysis. $J$ Glob Health. 2015;5(2):1-7. doi:10.7189/jogh.05-020415

3. Fang L, Gao P, Bao H, et al. Chronic obstructive pulmonary disease in China: a nationwide prevalence study. Lancet Respir Med. 2018;6 (6):421-430. doi:10.1016/s2213-2600(18)30103-6

4. Rehman AU, Hassali MAA, Muhammad SA, et al. The economic burden of chronic obstructive pulmonary disease (COPD) in the USA, Europe, and Asia: results from a systematic review of the literature. Expert Rev Pharmacoecon Outcomes Res. 2019:1-12. doi:10.1080/14737167.2020.1678385.

5. Miravitlles M, Ribera A. Understanding the impact of symptoms on the burden of COPD. Respir Res. 2017;18(67):1-11. doi:10.1186/ s12931-017-0548-3

6. Joshi M, Joshi A, Bartter T. Symptom burden in chronic obstructive pulmonary disease and cancer. Curr Opin Pulm Med. 2012;18 (2):97-103. doi:10.1097/MCP.0b013e32834fa84c

7. Tsiligianni I, Kocks JWH. Daytime symptoms of chronic obstructive pulmonary disease: a systematic review. NPJ Prim Care Respir Med. 2020;30(1):1-9. doi:10.1038/s41533-020-0163-5

8. Raghavan D, Varkey A, Bartter T. Chronic obstructive pulmonary disease: the impact of gender. Curr Opin Pulm Med. 2017;23 (2):117-123. doi:10.1097/mcp.0000000000000353

9. DeMeo DL, Ramagopalan S, Kavati A, et al. Women manifest more severe COPD symptoms across the life course. Int J Chron Obstruct Pulmon Dis. 2018;13:3021-3029. doi:10.2147/copd.s160270

10. Mahler DA. Mechanisms and measurement of dyspnea in chronic obstructive pulmonary disease. Proc Am Thorac Soc. 2006;3 (3):234-238. doi:10.1513/pats.200509-103SF

11. Jones PW, Harding G, Berry P, et al. Development and first validation of the COPD assessment test. Eur Respir J. 2009;34(3):648-654. doi:10.1183/09031936.00102509

12. Liu XC, Tang MQ. Reliability and validity of the pittsburgh sleep quality index. Chin J Catal. 1996;29(2):103-107.

13. Al-shair K, Kolsum U, Berry P, et al. Development, dimensions, reliability and validity of the novel Manchester COPD fatigue scale. Thorax. 2009;64(11):950-955. doi:10.1136/thx.2009.118109

14. Yang L, Bao L, Zhou Q, et al. Reliability and validity of Chinese edmonton frailty assessment scale. Int $J$ Endocrinol. 2016;37 (3):135-137.

15. Chen YH. Interpretation of global initiative for chronic obstructive lung disease global strategy for the diagnosis, management, and prevention of chronic obstructive pulmonary disease 2018 report. Chin J Front Med Sci. 2017;13(11):15-22.
16. Kroenke K, Spitzer RL, Williams JBW. The Patient Health Questionnaire-2: validity of a two-item depression screener. Med Care. 2003;41(11):1284-1292. doi:10.1097/01.mlr.0000093487.78664.3c

17. Goktalay T, Durmaz F, Havlucu Y, et al. Relation between sleep quality and quality of life in COPD patients: preliminary results. Chest. 2012;142(4):713A. doi:10.1378/chest.1365246

18. Al-shair K, Kolsum U, Singh D, et al. The effect of fatigue and fatigue intensity on exercise tolerance in moderate COPD. Lung. 2016;194(6):889-895. doi:10.1007/s00408-016-9931-y

19. Ban W. The relationship between insomnia and the COPD assessment test: a single-center cross-sectional study. Chest. 2017;152(4): A787. doi:10.1016/j.chest.2017.08.818

20. Yang L, Jiang Y, Xu S, et al. Evaluation of frailty status among older people living in urban communities by Edmonton Frail Scale in Wuhu, China: a cross-sectional study. Contemp Nurse. 2018;54 (6):630-639. doi:10.1080/10376178.2018.1552525

21. Schuler M, Strohmayer M, Mühlig S, et al. Assessment of depression before and after inpatient rehabilitation in COPD patients: psychometric properties of the German version of the Patient Health Questionnaire (PHQ-9/PHQ-2). J Affect Disord. 2018;232:168-275. doi:10.1016/j.jad.2018.02.037

22. Plummer F, Manea L, Trepel D, et al. Screening for anxiety disorders with the GAD-7 and GAD-2: a systematic review and diagnostic metaanalysis. Gen Hosp Psychiatry. 2016;39(3):24-31. doi:10.1016/ j.genhosppsych.2015.11.005

23. Burge S, Wedzicha JA. COPD exacerbations: definitions and classifications. Eur Respir J. 2003;21(Supplement 41):46S-53S. doi:10.1183/09031936.03.00078002

24. Wood WA, Chai X, Weisdorf D, et al. Comorbidity burden in patients with chronic GVHD. Bone Marrow Transpl. 2013;48(11):1429-1436. doi:10.1038/bmt.2013.70

25. Hurtig-Wennlof A, Hagstromer M, Olsson LA. The international physical activity questionnaire modified for the elderly: aspects of validity and feasibility. Public Health Nutr. 2010;13(11):1847-1854. doi:10.1017/s1368980010000157

26. Nunnally JC. Psychometric theory. Am Educ Res J 1978;5(3):83. doi: $10.2307 / 1161962$

27. Schweizer K. On the changing role of Cronbach's alpha in the evaluation of the quality of a measure. Eur J Psychol Assess. 2011;27(3):143-144. doi:10.1027/1015-5759/a000069

28. Torressánchez I, Valenza MC, Floresbarba MJ, et al. Comparison of fatigue and sleep quality between COPD and healthy subjects. Eur Respir J. 2013;42(57):1306S.

29. Limpawattana P, Putraveephong S, Inthasuwan P, et al. Frailty syndrome in ambulatory patients with COPD. Int $J$ Chron Obstruct Pulmon Dis. 2017;12:1193-1198. doi:10.2147/copd.S134233

30. de Negreiros Nobrega PV, Cavalcanti Maciel AC, de Almeida Holanda CM, et al. Sleep and frailty syndrome in elderly residents of long-stay institutions: a cross-sectional study. Geriatr Gerontol Int. 2014;14(3):605-612. doi:10.1111/ggi.12144

31. Foley D, Ancoli-Israel S, Britz P, et al. Sleep disturbances and chronic disease in older adults - results of the 2003 national sleep foundation sleep in america survey. J Psychosom Res. 2004;56 (5):497-502. doi:10.1016/j.jpsychores.2004.02.010

32. Ancoli-Israel S, Moore PJ, Jones V. The relationship between fatigue and sleep in cancer patients: a review. Eur $J$ Cancer Care (Engl). 2002;10(4):245-255. doi:10.1046/j.1365-2354.20 01.00263.x

33. Ensrud KE, Ewing SK, Taylor BC, et al. Frailty and risk of falls, fracture, and mortality in older women: the study of osteoporotic fractures. J Gerontol a Biol Sci Med Sci. 2007;62(7):744-751. doi:10.1093/gerona/62.7.744

34. Chervin RD. Sleepiness, fatigue, tiredness, and lack of energy in obstructive sleep apnea. Chest. 2000;118(2):372-379. doi:10.1378/ chest.118.2.372 
35. Van Cauter E, Spiegel K, Tasali E, Leproult R. Metabolic consequences of sleep and sleep loss. Sleep Med. 2008;9(supply1):s23s28. doi:10.1016/S1389-9457(08)70013-3

36. Piovezan RD, Abucham J, Thomatieli Dos Santos RV, et al. The impact of sleep on age-related sarcopenia: possible connections and clinical implications. Ageing Res Rev. 2015;23:210-220. doi:10.1016/ j.arr.2015.07.003

37. Landi F, Calvani R, Cesari M, et al. Sarcopenia as the biological substrate of physical frailty. Clin Liver Dis. 2015;19(3):367-374. doi:10.1016/j.cger.2015.04.005
38. Koo H-K, Jeong I, Lee SW, et al. Prevalence of chronic cough and possible causes in the general population based on the Korean national health and nutrition examination survey. Medicine. 2016;95 (37):1-7. doi:10.1097/md.0000000000004595

39. Sumner H, Woodcock A, Kolsum U, et al. Predictors of objective cough frequency in chronic obstructive pulmonary disease. Am J Respir Crit Care Med. 2013;187(9):943-949. doi:10.1164/rccm.201211-20000C

40. Rai DK, Kumar A, Anshuman A, et al. Phenotypic difference between smoker and non-smoker chronic obstructive pulmonary disease. JEMDS. 2017;6(30):2442-2445. doi:10.14260/Jemds/2017/527

\section{Publish your work in this journal}

The International Journal of COPD is an international, peer-reviewed journal of therapeutics and pharmacology focusing on concise rapid reporting of clinical studies and reviews in COPD. Special focus is given to the pathophysiological processes underlying the disease, intervention programs, patient focused education, and self management protocols. This journal is indexed on PubMed Central, MedLine and CAS. The manuscript management system is completely online and includes a very quick and fair peer-review system, which is all easy to use. Visit http://www.dovepress.com/testimonials.php to read real quotes from published authors. 